\title{
Positive solutions of critical semilinear problems involving a sublinear term at the origin
}

Elves A. B. Silva, João C. N. Pádua,

Departamento de Matemática, Universidade de Brasilia, 70910-900 Brasilia-DF, Brazil

E-mail: elves@mat.unb.br,jpadua@mat.unb.br

Sérgio H. M. Soares

Departamento de Matemática, Instituto de Ciências Matemáticas e de Computação, Universidade de São Paulo - Campus de São Carlos, Caixa Postal 668, 13560-970 São Carlos SP, Brazil

E-mail: monari@icmc.usp.br

By using variational methods we prove that a class of critical semilinear problems with a sublinear term at the origin possesses at least two positive solutions under conditions which allow the primitive of the sublinear term to assume negative values and it does not need to be convex. These problems are treated on bounded domains in $\mathbb{R}^{N}, N \geq 2$. October, 2004 ICMC-USP

\section{INTRODUCTION}

In this paper we first prove the existence of two positive solutions for the problem

$$
-\Delta u=|u|^{p-1} u+\lambda f(x, u) \text { in } \Omega, \quad u=0 \text { on } \partial \Omega,
$$

where $\Omega$ is a smooth bounded domain in $\mathbb{R}^{N}, N \geq 3, p=\frac{N+2}{N-2}$ is the critical Sobolev exponent, $\lambda>0$ is a real parameter, and the nonlinearity $f \in C(\bar{\Omega} \times \mathbb{R}, \mathbb{R})$ satisfies $f(x, 0) \equiv 0$ and the conditions:

$\left(f_{1}\right)$ there are constants $a_{1}, a_{2}>0$ and $0 \leq \sigma<p$ such that

$$
|f(x, s)| \leq a_{1}|s|^{\sigma}+a_{2}, \forall x \in \Omega, s \geq 0,
$$

$\left(f_{2}\right) \lim _{s \rightarrow 0^{+}} \frac{f(x, s)}{s}=\infty$, uniformly on $\bar{\Omega}$,

$\left(f_{3}\right)$ there is a constant $a>0$ such that $\liminf _{s \rightarrow \infty} \frac{F(x, s)}{s}>a$, uniformly on $\bar{\Omega}$, where $F(x, s)=\int_{0}^{s} f(x, t) d t$, 
$\left(f_{4}\right)$ there is a constant $\rho>0$ such that $f: \Omega \times[0, \rho] \rightarrow \mathbb{R}$ is nondecreasing with respect to the second variable.

Our first result is:

Theorem 1.1. Suppose $f$ satisfies $f(x, 0) \equiv 0$ and $\left(f_{1}\right)-\left(f_{4}\right)$. Then there exists $\lambda^{*}>0$ such that problem (1) possesses at least two positive solutions for every $\lambda \in\left(0, \lambda^{*}\right)$.

We recall that the conditions $\left(f_{1}\right)-\left(f_{4}\right)$ allow $f(x, s)$ to have an oscillatory behavior with respect to the variable $s$, the primitive $F(x, s)$ may assume negative values and does not need to be convex on the interval $(0, \infty)$. Theorem 1.1 establishes, in particular, the existence of two positive solutions for the following model problem

$$
-\Delta u=|u|^{p-1} u+\lambda(\varepsilon+\sin u) u^{q} \text { in } \Omega, \quad u=0 \text { on } \partial \Omega,
$$

where $0<\varepsilon$ and $0<q<1$.

In the seminal work [3], Ambrosetti-Brezis-Cerami considered problem (1) when $f(x, s) \equiv$ $s^{q}, 0<q<1$, and proved that there is $\Lambda>0$ such that (1) has at least two positive solutions for every $\lambda \in(0, \Lambda)$, one positive solution for $\lambda=\Lambda$ and no one for $\lambda>\Lambda$. Since then related results have been obtained, most of them for the p-Laplacian operator, as can be seen in $[5,6,8,12,15,16,23]$, and references therein.

When $\Omega$ is a bounded domain in $\mathbb{R}^{2}$ with smooth boundary $\partial \Omega$, we consider the class of problems

$$
-\Delta u=h(x, u)+\lambda f(x, u) \text { in } \Omega, \quad u=0 \text { on } \partial \Omega,
$$

where $\lambda>0$ is a real parameter. The natural growth restriction on the functions $f$ and $h$ is given by the inequality of Trudinger and Moser [18, 22]. More precisely (see [1, 14, 21]), we say that a function $g$ has a critical growth (subcritical growth) if there is $\alpha_{0}>0\left(\alpha_{0}=0\right)$ such that

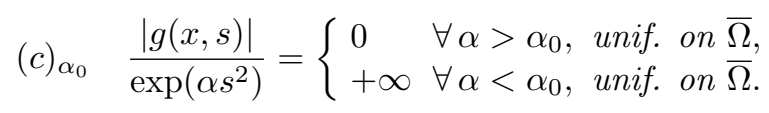

For problem (3) we assume that $h \in C(\bar{\Omega} \times \mathbb{R}, \mathbb{R})$ has critical growth and $f \in C(\bar{\Omega} \times \mathbb{R}, \mathbb{R})$ a subcritical one; $h(x, 0) \equiv 0, f(x, 0) \equiv 0$. Moreover, considering $H(x, s)=\int_{0}^{s} h(x, t) d t$ and denoting by $\lambda_{1}>0$ the first eigenvalue of $-\Delta$ on $H_{0}^{1}(\Omega)$, we assume

$\left(h_{1}\right) \limsup _{s \rightarrow 0^{+}} \frac{2 H(x, s)}{s^{2}}<\lambda_{1}$, uniformly on $\bar{\Omega}$,

$\left(h_{2}\right)$ for any $\theta>2$, there exists $R(\theta)>0$ such that

$$
0<\theta H(x, s) \leq \operatorname{sh}(x, s) \quad \forall x \in \Omega, s \geq R(\theta)
$$

$\left(h_{3}\right) \lim _{s \rightarrow \infty} \frac{\operatorname{sh}(x, s)}{\exp \left(\alpha_{0} s^{2}\right)}=\infty$, uniformly on $\bar{\Omega}$, 
$\left(h_{4}\right)$ there is a constant $\rho>0$ such that $h: \Omega \times[0, \rho] \rightarrow \mathbb{R}$ is nondecreasing with respect to the second variable.

Now we are ready to state our existence result for the case $N=2$.

THEOREM 1.2. Suppose $h$ satisfies $\left(h_{1}\right)-\left(h_{4}\right)$ and $(c)_{\alpha_{0}}$, with $\alpha_{0}>0$, and $f$ satisfies $\left(f_{2}\right),\left(f_{3}\right),\left(f_{4}\right)$ and $(c)_{\alpha_{0}}$, with $\alpha_{0}=0$. Then there exists $\lambda^{*}>0$ such that (3) possesses at least two positive solutions for every $\lambda \in\left(0, \lambda^{*}\right)$.

We observe that this last result guarantees the existence of two positive solutions for the problem

$$
-\Delta u=\mu u \exp \left(u^{2}\right)+\lambda(\varepsilon+\sin u) u^{q} \exp (u) \text { in } \Omega, u=0 \text { on } \partial \Omega
$$

with $0<\varepsilon, 0<\mu<\lambda_{1}$ and $0<q<1$.

Related results about problems with exponential growth can be seen in $[1,2,7,14,21]$ and the references therein.

In [21], Silva and Soares showed that problem (3) with critical growth possesses at least two nontrivial solutions provided the nonlinearity $H+\lambda F$ satisfies a condition of convexity with respect to variable $s$. We observe that Theorem 1.2 improves this result when $N=2$, since $\left(h_{1}\right)-\left(h_{3}\right)$ and $\left(f_{2}\right)-\left(f_{4}\right)$ allow $g(x, s)=h(x, s)+\lambda f(x, s)$ to have an oscillatory behavior on the variable $s$.

In order to prove Theorems 1.1 and 1.2, we first establish the existence of a nontrivial local minimum for the associated functional. Then we use an argument of Brezis and Nirenberg [10] to verify that the associated functional satisfies the Palais-Smale condition on a given interval of the real line, under the assumption that the functional has only the zero and the local minimum as critical points. Since now the primitive $F(x, s)$ may not be convex on the interval $(0, \infty)$, we first get an estimate from below for the term $F(x, s+t)-F(x, s)-g(x, s) t$, when $s$ and $t$ are small (see Lemma 2.2). Then this estimate is used to prove that the level associated with the Mountain Pass Theorem belongs to that

interval; then we use the celebrated Theorem of Ambrosetti and Rabinowitz [4] to find the second nontrivial solution.

\section{PROOF OF THEOREM 1.1}

Here we consider the Sobolev space $H_{0}^{1}(\Omega)$ with its usual norm. We assume that $f(x, s) \equiv$ 0 when $s \leq 0$ without changing the symbols $f$ and $F$. I order to prove Theorem 1.1 it suffices to show that there is $\lambda^{*}$ such that the problem

$$
-\Delta u=\left(u^{+}\right)^{p}+\lambda f(x, u) \text { in } \Omega, \quad u=0 \text { on } \partial \Omega,
$$


possesses at least two nontrivial solutions for each $\lambda \in\left(0, \lambda^{*}\right)$. The critical points of the associated $C^{1}$ functional $I_{\lambda}: H_{0}^{1}(\Omega) \rightarrow \mathbb{R}$, given by

$$
I_{\lambda}(u)=\frac{1}{2} \int_{\Omega}|\nabla u|^{2} d x-\frac{1}{p+1} \int_{\Omega}\left(u^{+}\right)^{p+1} d x-\lambda \int_{\Omega} F(x, u) d x,
$$

are precisely the solutions of problem (4). If $u$ denotes one of these solutions, then $I_{\lambda}^{\prime}(u)\left(u^{-}\right)=0$; thus $u \geq 0$. To see that $u>0$ in $\Omega$ by the strong maximum principle, we observe that $u$ is also a solution of the problem

$$
-\Delta u+c u=\left(u^{+}\right)^{p}+\lambda f(x, u)+c u^{+} \text {in } \Omega, \quad u=0 \text { on } \partial \Omega,
$$

where $c \geq 0$ is such that the term on the right is nonnegative for $x$ in $\Omega$; the existence of this $c$ follows from $\left(f_{1}\right)-\left(f_{2}\right), p>\max (1, \sigma)$ and the continuity of $\left(s^{p}+\lambda f(x, s)\right) / s$ on a compact subset of $\bar{\Omega} \times(0, \infty)$.

The next result gives the first solution for problem (4).

Proposition 2.1. Suppose $f \in C(\bar{\Omega} \times \mathbb{R}, \mathbb{R})$ satisfies $\left(f_{1}\right)$ and $\left(f_{2}\right)$. Then there exists $\lambda^{*}>0$ such that problem (4) possesses at least one positive solution $u_{\lambda}$ for every $\lambda \in\left(0, \lambda^{*}\right)$. Moreover, $u_{\lambda}$ is a local minimum of $I_{\lambda}$ and $I_{\lambda}\left(u_{\lambda}\right)<0$.

Proof: By $\left(f_{1}\right)$, the functional $J: H_{0}^{1}(\Omega) \rightarrow \mathbb{R}$, given by $J(u)=\int_{\Omega} F(x, u) d x$ is continuous. Thus, given $\varepsilon>0$, there is $\rho_{1}>0$ such that $|J(u)|<\varepsilon$ if $\|u\| \leq \rho_{1}$. Then,

$$
I_{\lambda}(u) \geq\left(\frac{1}{2}-\frac{1}{p+1} S^{(p+1) / 2}\|u\|^{p-1}\right)\|u\|^{2}-\lambda \varepsilon,
$$

where $S$ denotes the best Sobolev constant.

Let $\rho \in\left(0, \rho_{1}\right)$ be such that

$$
I_{\lambda}(u) \geq \frac{1}{4}\|u\|^{2}-\lambda \varepsilon \text { if }\|u\| \leq \rho .
$$

Setting $\lambda^{*}=\rho^{2} / 8 \varepsilon$ we obtain

$$
I_{\lambda}(u) \geq \rho^{2} / 8 \text { if }\|u\|=\rho \text { and } I_{\lambda}(u) \geq-\rho^{2} / 8 \text { if }\|u\| \leq \rho
$$

for every $0<\lambda<\lambda^{*}$. Let $c_{\lambda}$ stands for the infimum of $I_{\lambda}$ on $A=\overline{B_{\rho}(0)}$. From $\left(f_{2}\right)$, given $\phi \in H_{0}^{1}(\Omega), \phi \neq 0, \phi \geq 0$, there exists $t=t(\phi)>0$ sufficiently small such that $I_{\lambda}(t \phi)<0$. Then,

$$
\inf _{u \in A} I_{\lambda}(u)<0<\inf _{u \in \partial A} I_{\lambda}(u) .
$$

Therefore, by Ekeland's variational principle [13], there is a sequence $\left(u_{n}\right) \subset \operatorname{int}(A)$ such that

$$
\left\|I_{\lambda}^{\prime}\left(u_{n}\right)\right\| \rightarrow 0 \quad \text { and } \quad I_{\lambda}\left(u_{n}\right) \rightarrow c_{\lambda} .
$$

Publicado pelo ICMC-USP Sob a supervisão da $\mathrm{CPq} / \mathrm{ICMC}$ 
As $\left(u_{n}\right)$ is bounded and $A$ is a closed convex set, we can suppose that there exist $u_{\lambda} \in A \subset$ $H_{0}^{1}(\Omega)$ and a subsequence of $\left(u_{n}\right)$, still denoted by $\left(u_{n}\right)$, such that

$$
\left\{\begin{array}{l}
u_{n} \rightarrow u_{\lambda} \text { weakly in } H_{0}^{1}(\Omega), \\
u_{n} \rightarrow u_{\lambda} \text { strongly in } L^{r}(\Omega), \text { for every } 1 \leq r<p+1, \\
u_{n} \rightarrow u_{\lambda} \text { a.e. in } \Omega,
\end{array}\right.
$$

Moreover, the sequence $\left(f\left(x, u_{n}(x)\right)\right)$ converges to $f\left(x, u_{\lambda}(x)\right)$ a.e. in $\Omega$ and by $\left(f_{1}\right)$ one deduces it is bounded in $L^{\frac{p+1}{p}}$. Hence this sequence converges weakly to $f\left(x, u_{\lambda}\right)$; similarly, $\left(u_{n}^{+}\right)^{p} \rightarrow\left(u_{\lambda}^{+}\right)^{p}$. Consequently, passing to the limit in $I_{\lambda}^{\prime}\left(u_{n}\right) v$, as $n \rightarrow \infty$, we have

$$
\int_{\Omega} \nabla u_{\lambda} \nabla v d x-\int_{\Omega}\left(u_{\lambda}^{+}\right)^{p} v d x-\lambda \int_{\Omega} f\left(x, u_{\lambda}\right) v d x=0, \forall v \in H_{0}^{1}(\Omega),
$$

and thus $u_{\lambda}$ is a critical point of the functional $I_{\lambda}$. On the other hand, from $\left(f_{1}\right)$ and Fatou's Lemma, we have

$$
\begin{aligned}
c_{\lambda} & \geq \liminf _{n \rightarrow \infty}\left[I_{\lambda}\left(u_{n}\right)-\frac{1}{p+1}\left\langle I_{\lambda}^{\prime}\left(u_{n}\right), u_{n}\right\rangle\right] \\
& =\liminf _{n \rightarrow \infty}\left[\frac{1}{N}\left\|u_{n}\right\|^{2}+\lambda \int_{\Omega}\left(\frac{1}{p+1} f\left(x, u_{n}\right) u_{n}-F\left(x, u_{n}\right)\right) d x\right] \\
& \geq \frac{1}{N}\left\|u_{\lambda}\right\|^{2}+\lambda \int_{\Omega}\left(\frac{1}{p+1} f\left(x, u_{\lambda}\right) u_{\lambda}-F\left(x, u_{\lambda}\right)\right) d x \\
& =I_{\lambda}\left(u_{\lambda}\right)-\frac{1}{p+1}\left\langle I_{\lambda}^{\prime}\left(u_{\lambda}\right), u_{\lambda}\right\rangle=I_{\lambda}\left(u_{\lambda}\right) \geq c_{\lambda} .
\end{aligned}
$$

Thus, $I_{\lambda}\left(u_{\lambda}\right)=c_{\lambda}<0$. Therefore, by (7), the function $u_{\lambda}$ is a nontrivial solution of (4) and is in the interior of $B_{\rho}$. This completes the proof of the proposition.

Now we prove that $I_{\lambda}$ satisfies $(\mathrm{PS})_{c}$ below a given level, whenever we suppose that zero and $u_{\lambda}$ are the unique critical points (see $\left.[3,10]\right)$.

Lemma 2.1. Suppose $f$ satisfies $\left(f_{1}\right)-\left(f_{4}\right)$. If zero and $u_{\lambda}$ are the unique critical points of $I_{\lambda}$, then $I_{\lambda}$ satisfies $(P S)_{c}$ condition for every $c<\bar{c} \equiv I_{\lambda}\left(u_{\lambda}\right)+\frac{1}{N} S^{\frac{N}{2}}$ and $0<\lambda<\lambda^{*}$.

Proof: Let $\left(u_{n}\right)$ be a sequence in $H_{0}^{1}(\Omega)$ such that

$$
I_{\lambda}\left(u_{n}\right) \rightarrow c<\bar{c} \text { and } \quad I_{\lambda}^{\prime}\left(u_{n}\right) \rightarrow 0 .
$$

We observe that $g(x, s) \equiv\left(s^{+}\right)^{p}+\lambda f(x, s)$ satisfies Ambrosetti-Rabinowitz condition

$$
0 \leq \mu G(x, s) \leq s g(x, s) \text { for all }|s| \geq M,
$$

and for some $\mu>2$. Therefore, the sequence $\left(u_{n}\right)$ is bounded in $H_{0}^{1}(\Omega)$ (see $[4,20]$ ). Thus, just as in Proposition 2.1, there exists a subsequence of $\left(u_{n}\right)$, still denoted by $\left(u_{n}\right)$, 
which converges to a weak solution of problem (4). Next we use an argument due to Brezis-Nirenberg [10] to show the convergence of a subsequence of $\left(u_{n}\right)$ in $H_{0}^{1}(\Omega)$. We set $v_{n}=u_{n}-u$, then apply a result of Brezis-Lieb [9] to the sequence $\left(u_{n}^{+}\right)^{p+1}$ and use the condition $\left(f_{1}\right)$ and the fact that $u$ is a solution of (4) to obtain

$$
\int\left|\nabla v_{n}\right|^{2}=\int\left(v_{n}^{+}\right)^{p+1}+o(1) \quad \text { and } \quad I_{\lambda}(u)+\frac{1}{N} \int\left|\nabla v_{n}\right|^{2}=c+o(1) .
$$

Hence, for a subsequence of $\left(v_{n}\right)$ one gets

$$
\int\left|\nabla v_{n}\right|^{2} \rightarrow k \geq 0 \quad \text { and } \quad \int\left(v_{n}^{+}\right)^{p+1} \rightarrow k
$$

It follows from Sobolev imbedding that $k \geq S k^{2 /(p+1)}$, which imply either $k=0$ or $k \geq$ $S^{N / 2}$. If either $u=0$ or $u=u_{\lambda}$ then $k=0$, since otherwise we would have either

$$
\frac{1}{N} S^{N / 2} \leq c \text { or } I_{\lambda}\left(u_{\lambda}\right)+\frac{1}{N} S^{N / 2} \leq c
$$

respectively. Hence the condition $(\mathrm{PS})_{c}$ holds for each $c<\bar{c}$.

In order to find the appropriate level to the use of Mountain Pass Theorem we follow the method of [10] (see also [3]). However, under our assumptions the lower order perturbation need not be increasing, so we start by proving an elementary estimate which will play a significant role in the sequel.

Lemma 2.2. Suppose $f \in C(\bar{\Omega} \times \mathbb{R}, \mathbb{R})$ satisfies $f(x, 0) \equiv 0,\left(f_{3}\right)$ and $\left(f_{4}\right)$. Then, given $\gamma>0$ there exist constants $C>0$ and $r>0$ such that

$$
F(x, s+t)-F(x, s)-f(x, s) t \geq-C t^{\gamma},
$$

for every $x \in \Omega, 0 \leq s \leq r, t \geq 0$.

Proof: By $\left(f_{3}\right)$, we have $s_{0}>\rho$ such that

$$
F(x, s) \geq a s>0, \forall x \in \Omega, s \geq s_{0} .
$$

Now, invoking $f(x, 0) \equiv 0$ and $f \in C(\bar{\Omega} \times \mathbb{R}, \mathbb{R})$, we find $r \in(0, \rho / 2)$ such that

$$
f(x, s) \leq \frac{a}{2}, \forall x \in \Omega, 0 \leq s \leq r .
$$

Hence, for every $x \in \Omega, s \leq r$, we get

$$
\int_{s}^{s_{0}}|f(x, \tau)-f(x, s)| d \tau \leq\left[\frac{a}{2}+K\right] s_{0} \doteq c_{1},
$$

Publicado pelo ICMC-USP 
where $K$ denotes the maximum of $|f(x, s)|$ for $x$ in $\bar{\Omega}$ and $0 \leq s \leq s_{0}$. Consequently, by (11)-(13), we have

$$
F(x, s+t)-F(x, s)-f(x, s) t=\int_{s}^{t+s}[f(x, \tau)-f(x, s)] d \tau \geq-c_{1}+\frac{a}{2} t-\left\|F\left(., s_{0}\right)\right\|_{\infty}
$$

whenever $x \in \bar{\Omega}, 0 \leq s \leq r, s_{0} \leq t$. Therefore, there exists $R>0$ such that

$$
F(x, s+t)-F(x, s)-f(x, s) t \geq 0, \quad \forall x \in \bar{\Omega}, \quad 0 \leq s \leq r, \quad R \leq t .
$$

On the other hand, given $x \in \Omega$ and $0 \leq s, t \leq r$, we find $\theta \in(0,1)$ such that

$$
F(x, s+t)-F(x, s)-f(x, s) t=[f(x, s+\theta t)-f(x, s)] t .
$$

Thus, by $r<\rho / 2$ and $\left(f_{2}\right)$, we get

$$
F(x, s+t)-F(x, s)-f(x, s) t \geq 0, \forall x \in \bar{\Omega}, 0 \leq s, t \leq r .
$$

Next, we use $f \in C(\bar{\Omega} \times \mathbb{R}, \mathbb{R})$ to obtain $M>0$ such that

$$
F(x, s+t)-F(x, s)-f(x, s) t \geq-M, \forall x \in \bar{\Omega}, 0 \leq s \leq r, r \leq t \leq R .
$$

Finally, choosing $C>0$ such that $C r^{\gamma} \geq M$, we invoke (14)-(16) to conclude that (10) holds. The lemma is proved.

The next auxiliary lemma is in [16].

Lemma 2.3. If $r>2$, then for any $\sigma \in(1, r-1)$, there exists a constant $C>0$ such that

$$
(1+t)^{r} \geq 1+t^{r}+r t+r t^{r-1}-C t^{\sigma}, \quad \forall t \geq 0
$$

In order to use the Mountain Pass Theorem we need the following estimate.

Lemma 2.4. Suppose $f$ satisfies $\left(f_{1}\right)-\left(f_{4}\right)$. Then, for every $\lambda \in\left(0, \lambda^{*}\right)$, there exists $\varepsilon>0$ such that

$$
\max \left\{I_{\lambda}\left(u_{\lambda}+t v_{\varepsilon}\right) \mid t \geq 0\right\}<I_{\lambda}\left(u_{\lambda}\right)+\frac{1}{N} S^{\frac{N}{2}} .
$$

Proof Let $r>0$ be given by Lemma 2.2 for $\gamma=(N+1) /(N-2)$. Since $u_{\lambda}>0$ and $u_{\lambda} \in C^{1, \alpha}(\bar{\Omega}) \cap H_{0}^{1}(\Omega)$, we obtain $x_{0} \in \Omega$ and $R, r_{0}>0$ such that $B_{2 R}\left(x_{0}\right) \subset \Omega$ and

$$
0<r_{0} \leq u_{\lambda}(x) \leq r, \forall x \in B_{2 R}\left(x_{0}\right) .
$$


Without loss of generality we may assume that $x_{0}=0$ and we take $\phi \in \mathcal{D}\left(B_{2 R}(0)\right)$ such that $0 \leq \phi(x) \leq 1$ in $B_{2 R}(0)$ and $\phi(x) \equiv 1$ on $B_{R}(0)$. Set

$$
u_{\varepsilon}(x)=\frac{\phi(x)}{\left(\varepsilon+|x|^{2}\right)^{\frac{N-2}{2}}} \text { and } v_{\varepsilon}(x)=\frac{u_{\varepsilon}(x)}{\left\|u_{\varepsilon}\right\|_{p+1}}, \varepsilon>0 .
$$

Then, $v_{\varepsilon}$ satisfies as $\varepsilon \rightarrow 0($ see $[10,12])$

$$
\begin{aligned}
\left\|\nabla v_{\varepsilon}\right\|_{2}^{2} & =S+O\left(\varepsilon^{\frac{N-2}{2}}\right) \\
\left\|v_{\varepsilon}\right\|_{\tau}^{\tau} & =K \varepsilon^{\frac{N(2-\tau)+2 \tau}{4}}, \quad \text { if } \tau>\frac{N}{N-2} .
\end{aligned}
$$

We first apply Lemma 2.3 to the term $\left|u_{\lambda}+t v_{\varepsilon}\right|^{p+1}$ of $I_{\lambda}\left(u_{\lambda}+t v_{\varepsilon}\right)$, with $\sigma=\frac{N+1}{N-2}$, then we use $I_{\lambda}^{\prime}\left(u_{\lambda}\right)\left(t v_{\varepsilon}\right)=0$, to obtain

$$
\begin{aligned}
I_{\lambda}\left(u_{\lambda}+t v_{\varepsilon}\right) & \leq I_{\lambda}\left(u_{\lambda}\right)+\frac{t^{2}}{2} \int_{\Omega}\left|\nabla v_{\varepsilon}\right|^{2} d x-\frac{t^{p+1}}{p+1} \\
& -t^{p} \int_{\Omega} v_{\varepsilon}^{p} u_{\lambda} d x+C t^{\frac{N+1}{N-2}} \int_{\Omega} u_{\lambda}^{\frac{N-1}{N-2}} v_{\varepsilon}^{\frac{N+1}{N-2}} d x \\
& -\lambda \int_{\Omega}\left(F\left(x, u_{\lambda}+t v_{\varepsilon}\right)-F\left(x, u_{\lambda}\right)-f\left(x, u_{\lambda}\right) t v_{\varepsilon}\right) d x .
\end{aligned}
$$

Using Lemma 2.2 with $\gamma=\frac{N+1}{N-2}$ and (17) in the last term, one has

$$
\begin{aligned}
I_{\lambda}\left(u_{\lambda}+t v_{\varepsilon}\right) & \leq I_{\lambda}\left(u_{\lambda}\right)+\frac{t^{2}}{2} \int_{\Omega}\left|\nabla v_{\varepsilon}\right|^{2} d x-\frac{t^{p+1}}{p+1}-r_{0} t^{p} \int_{\Omega} v_{\varepsilon}^{p} d x \\
& +C(1+\lambda) r^{\frac{N-1}{N-2}} t^{\frac{N+1}{N-2}} \int_{\Omega} v_{\varepsilon}^{\frac{N+1}{N-2}} d x .
\end{aligned}
$$

By (18)-(19), there exist $\varepsilon_{0}>0$ and positive constants $C_{1}$ and $C_{2}$ such that

$$
I_{\lambda}\left(u_{\lambda}+t v_{\varepsilon}\right) \leq I_{\lambda}\left(u_{\lambda}\right)+\Psi_{\varepsilon}(t), \quad \forall \varepsilon \in\left(0, \varepsilon_{0}\right),
$$

where

$$
\Psi_{\varepsilon}(t)=\frac{S}{2} t^{2}-\frac{t^{p+1}}{p+1}+C_{1}\left[t^{2} \varepsilon^{\frac{N-2}{2}}+(1+\lambda) t^{\frac{N+1}{N-2}} \varepsilon^{\frac{N-1}{4}}\right]-C_{2} t^{p} \varepsilon^{\frac{N-2}{4}} .
$$

Since $\Psi_{\varepsilon}(t) \rightarrow 0$, as $t \rightarrow 0^{+}$, and $\Psi_{\varepsilon}(t) \rightarrow-\infty$, as $t \rightarrow+\infty$, there exists $t_{\varepsilon}>0$ such that $\Psi_{\varepsilon}\left(t_{\varepsilon}\right)=\max \left\{\Psi_{\varepsilon}(t) ; t \geq 0\right\}$. It follows from (6) that

$$
0<\frac{\rho^{2}}{8} \leq \max _{t \geq 0} I_{\lambda}\left(u_{\lambda}+t v_{\varepsilon}\right) \leq I_{\lambda}\left(u_{\lambda}\right)+\Psi_{\varepsilon}\left(t_{\varepsilon}\right)<\Psi_{\varepsilon}\left(t_{\varepsilon}\right) .
$$


Now, we claim that there are $T_{1}, T_{2}>0$ such that $t_{\varepsilon} \in\left[T_{1}, T_{2}\right]$ for every $\varepsilon \in\left(0, \varepsilon_{0}\right)$. In fact, the lower estimate for $t_{\varepsilon}$ follows from (20) since $\Psi_{\varepsilon}(t) \rightarrow 0$ as $t \rightarrow 0^{+}$. For the upper estimate, we assume by contradiction that there is a sequence $\left(\varepsilon_{n}\right)$, with $\varepsilon_{n} \rightarrow 0$, such that $t_{\varepsilon_{n}} \rightarrow+\infty$, as $n \rightarrow \infty$. By $\Psi_{\varepsilon}^{\prime}\left(t_{\varepsilon_{n}}\right)=0$, it follows that

$$
t_{\varepsilon_{n}}^{p}=t_{n}\left(S+2 C_{1} \varepsilon_{n}^{\frac{N-2}{2}}\right)+(1+\lambda) \frac{N+1}{N-2} C_{1} t_{\varepsilon_{n}}^{\frac{3}{N-2}}-C_{2} p t_{\varepsilon_{n}}^{p-1} \varepsilon_{n}^{\frac{N-2}{4}},
$$

which can not happen for $t_{\varepsilon_{n}}$ sufficiently large. This proves the claim.

Now, observing that the maximum of $p(t)=\frac{S}{2} t^{2}-\frac{t^{p+1}}{p+1}, t>0$, occurs in $t=S^{\frac{1}{p}}$, with maximum value $\frac{1}{N} S^{\frac{N}{2}}$, by (20) and the lower and upper estimates for $t_{\varepsilon}$, we have $C_{3}, C_{4}>0$ such that

$$
I_{\lambda}\left(u_{\lambda}+t v_{\varepsilon}\right) \leq I_{\lambda}\left(u_{\lambda}\right)+\frac{1}{N} S^{\frac{N}{2}}+\left\{C_{3}\left[\varepsilon^{\frac{N-2}{4}}+(1+\lambda) \varepsilon^{\frac{3}{4}}\right]-C_{4}\right\} \varepsilon^{\frac{N-2}{4}},
$$

for every $t \geq 0$. Since the expression inside the braces is negative for $\varepsilon>0$ sufficiently small, one has

$$
\max \left\{I_{\lambda}\left(u_{\lambda}+t v_{\varepsilon}\right) \mid t \geq 0\right\}<I_{\lambda}\left(u_{\lambda}\right)+\frac{1}{N} S^{\frac{N}{2}},
$$

provided $\varepsilon$ is sufficiently small. This concludes the proof of Lemma 2.4.

Now we are ready to complete the proof of Theorem 1.1. In fact, if $I_{\lambda}$ possesses two nontrivial critical points there is nothing to prove. Otherwise, we use (9) to choose $u_{1}$ such that $I_{\lambda}\left(u_{1}\right)<0$ and $\left\|u_{1}\right\|>\rho$, then we consider the family $\Gamma$ of all continuous paths $\gamma$ joining $u_{\lambda}$ to $u_{1}$ (see (6) and the proof of Proposition 2.1), and define

$$
\bar{c}_{\lambda}=\inf _{\gamma \in \Gamma} \max _{t \in[0,1]} I_{\lambda}(\gamma(t)) \geq \frac{\rho^{2}}{8} .
$$

By Lemmas 2.2 and 2.5, one can apply the Mountain Pass Theorem [4] to obtain a second nontrivial critical point of $I_{\lambda}$ at the level $\bar{c}_{\lambda}$. The proof of Theorem 1.1 is complete.

\section{PROOF OF THEOREM 1.2}

In order to treat problem (3) variationally in $H_{0}^{1}(\Omega)$, we use the inequalities of Trudinger and Moser (see [18], [22]), which provide

$$
\exp \left(\alpha u^{2}\right) \in L^{1}(\Omega), \forall u \in H_{0}^{1}(\Omega), \forall \alpha>0
$$

and

$$
\sup _{\|u\| \leq 1} \int_{\Omega} \exp \left(\alpha u^{2}\right) d x \leq C, \forall \alpha \leq 4 \pi, u \in H_{0}^{1}(\Omega) .
$$


Under condition $(c)_{\alpha_{0}}$, given $\beta>\alpha_{0}$, there exists $C=C(\beta)>0$ such that

$$
\max \{|f(x, s)|,|F(x, s)|,|h(x, s)|,|H(x, s)|\} \leq C \exp \left(\beta s^{2}\right),
$$

for every $x \in \bar{\Omega}$ and $s \geq 0$. Using these estimates, it can be proved that the critical points of the $C^{1}$ functional $I_{\lambda}: H_{0}^{1}(\Omega) \rightarrow \mathbb{R}$, given by

$$
I_{\lambda}(u)=\frac{1}{2} \int_{\Omega}|\nabla u|^{2} d x-\int_{\Omega} H(x, u) d x-\lambda \int_{\Omega} F(x, u) d x,
$$

are the solutions of (3). Actually, by regularity argument it can be proved that $u \in C^{1}(\bar{\Omega})$ (see $[11,17]$ ). We assume $f(x, s)=h(x, s)=0$, for every $x \in \Omega, s \leq 0$. We also observe that conditions $\left(h_{1}\right),\left(f_{1}\right)$, and the argument described in Section 2 imply that $u>0$ in $\Omega$.

The next auxiliary lemma is a result due to Lions [19], which will be essential to verify, via contradiction, that functional $I_{\lambda}$ satisfies $(P S)_{c}$, for $c$ below a given level.

LEMMA 3.1. Let $\left\{u_{n} \in H_{0}^{1}(\Omega) ;\left\|u_{n}\right\|=1\right\}$ be a sequence converging weakly to a nonzero function $u$. Then, for every $0<p<\left(1-\|u\|^{2}\right)^{-1}$, we have

$$
\sup _{n \in \mathbb{N}} \int_{\Omega} \exp \left(p 4 \pi u_{n}^{2}\right) d x<\infty .
$$

In our first result in this section, we establish the existence of a solution for (3) when $\lambda$ is sufficiently small.

Proposition 3.1. Suppose $h \in C(\bar{\Omega} \times \mathbb{R}, \mathbb{R})$ satisfies $\left(h_{1}\right),(c)_{\alpha_{0}}$, with $\alpha_{0}>0$, and $f \in C(\bar{\Omega} \times \mathbb{R}, \mathbb{R})$ satisfies $\left(f_{2}\right)$ and $(c)_{\alpha_{0}}$, with $\alpha_{0}=0$. Then, there exists $\lambda^{*}>0$ such that (3) possesses at least one positive solution $u_{\lambda}$ for each $\lambda \in\left(0, \lambda^{*}\right)$.

Proof: Taking $\beta>\alpha_{0}$, by (23), given $q>2$, there exist $s_{0}>0$ and a positive constant $C$ such that

$$
|H(x, s)| \leq C s^{q} \exp \left(\beta s^{2}\right), \forall s \geq s_{0} .
$$

Now using that $h$ is continuous and satisfies $\left(h_{1}\right)$, we can choose $0<\mu<\lambda_{1}$ such that

$$
|H(x, s)| \leq \frac{\mu}{2} s^{2}+C s^{q} \exp \left(\beta s^{2}\right), \forall x \in \Omega, s \in \mathbb{R},
$$

where $C$ is a positive constant. Combining this with Hölder's inequality we get

$$
\int_{\Omega}|H(x, u)| d x \leq \frac{\mu}{2 \lambda_{1}}\|u\|^{2}+C\left[\int_{\Omega} \exp \left(\beta r\|u\|^{2}\left(\frac{|u|}{\|u\|}\right)^{2} d x\right]^{1 / r}\|u\|_{s q}^{q},\right.
$$

where $r$ and $s$ are positive and $1 / r+1 / s=1$. Moreover, the functional

$$
J(u)=\int_{\Omega} F(x, u) d x
$$

Publicado pelo ICMC-USP Sob a supervisão da $\mathrm{CP}$ / / ICMC 
is continuous at $u=0$, thus for $\varepsilon>0$ there exists $\delta>0$ such that $|J(u)|<\varepsilon$ for $\|u\|<\delta$. Taking $\sigma>0$ sufficiently small so that $\beta r \sigma^{2}<4 \pi, \sigma \leq \delta$, by (22), (24) and Sobolev embedding we have

$$
I_{\lambda}(u) \geq \frac{1}{2}\left(1-\frac{\mu}{\lambda_{1}}\right)\|u\|^{2}-C\|u\|^{q}-\lambda \epsilon,
$$

for every $u \in H_{0}^{1}(\Omega)$ with $\|u\| \leq \sigma$. Since $q>2$, we can diminish $\sigma$ if necessary and then choose $a>0$ and $\lambda^{*}>0$ such that

$$
I_{\lambda}(u) \geq-\frac{a}{2} \sigma^{2} \quad \text { if } \quad\|u\| \leq \sigma \quad \text { and } \quad I_{\lambda}(u) \geq \frac{a}{2} \quad \text { if } \quad\|u\|=\sigma,
$$

for every $0<\lambda<\lambda^{*}$. Let $c_{\lambda}$ be the infimum of $I_{\lambda}$ on $A=\overline{B_{\sigma}(0)}$. Since $g(x, s) \doteq$ $h(x, s)+\lambda f(x, s)$ satisfies $\left(f_{2}\right)$, given $\phi \in \mathcal{D}(\Omega), \phi \neq 0, \phi \geq 0$, there exists $t=t(\phi)$ such that $I_{\lambda}(t \phi)<0$. Then

$$
c_{\lambda}=\inf _{u \in A} I_{\lambda}(u)<0<\inf _{u \in \partial A} I_{\lambda}(u) .
$$

Applying the Ekeland's variational principle, as in the proof of Proposition 2.1, there is a sequence $\left(u_{n}\right) \subset \operatorname{int}(A)$ such that

$$
\left\|I_{\lambda}^{\prime}\left(u_{n}\right)\right\| \rightarrow 0 \text { and } I\left(u_{n}\right) \rightarrow c_{\lambda} .
$$

Since $\left(u_{n}\right)$ is bounded and $A$ is a closed convex set, we can assume that there exist $u \in A$ and a subsequence of $\left(u_{n}\right)$, still denoted by $\left(u_{n}\right)$, converging weakly in $H_{0}^{1}(\Omega)$ to a solution $u_{\lambda}$ of (3). Moreover, we may assume that $u_{n}(x) \rightarrow u_{\lambda}(x)$, for almost every $x \in \Omega$, and that there exists a function $M=M(x)$ in $L^{p}(\Omega)$ such that $|u(x)| \leq M(x)$ for all $x$ in $\Omega$ and $p \geq 1$. Now we will show that $\left\|u_{n}\right\| \rightarrow\|u\|$ as $n \rightarrow \infty$. By condition $(c)_{\alpha_{0}}$, the inequality

$$
|h(x, s) s| \leq C|s| \exp \left(\beta s^{2}\right)
$$

holds for $x$ in $\Omega$ and $s$ in $\mathbb{R}$. As in (24), we use (22) and Hölder's inequality to obtain

$$
\int_{E}\left|h\left(x, u_{n}(x)\right) u_{n}(x)\right| d x \leq C \int_{E} M(x)^{s} d x,
$$

where $E$ is any measurable subset of $\Omega$. Thus, the set $\left\{h\left(x, u_{n}\right) u_{n}\right\}$ is uniformly integrable and Vitali's theorem imply that the sequence $\left(h\left(x, u_{n}\right) u_{n}\right)$ converges to $h\left(x, u_{\lambda}\right) u_{\lambda}$ in $L^{1}(\Omega)$; similarly, $f\left(x, u_{n}\right) u_{n} \rightarrow f\left(x, u_{\lambda}\right) u_{\lambda}$ in $L^{1}(\Omega)$ as $n \rightarrow \infty$. Now we use $(27)$ and the fact that $u_{\lambda}$ is a critical point of $I_{\lambda}$ to conclude that $\left\|u_{n}\right\| \rightarrow\left\|u_{\lambda}\right\|$ as $n \rightarrow \infty$; hence, the sequence $\left(u_{n}\right)$ converges to $u_{\lambda}$ in $H_{0}^{1}(\Omega)$ and $I_{\lambda}\left(u_{\lambda}\right)=c_{\lambda}$. Therefore, from (26), $I_{\lambda}$ possesses a nontrivial local minimum $u_{\lambda} \in \operatorname{int}(A)$. This completes the proof of Proposition 3.1.

Considering the solution $u_{\lambda}$ given by Proposition 3.1, we shall verify that $I_{\lambda}$ satisfies $(P S)_{c}$ condition below the level $I_{\lambda}\left(u_{\lambda}\right)+\frac{2 \pi}{\alpha_{0}}$, whenever we suppose that zero and $u_{\lambda}$ are the unique critical points of $I_{\lambda}$. 
Lemma 3.2. Suppose $h$ satisfies $\left(h_{2}\right)$ and $(c)_{\alpha_{0}}$, with $\alpha_{0}>0$, and $f$ satisfies $(c)_{\alpha_{0}}$, with $\alpha_{0}=0$. Assume that zero and $u_{\lambda}$ are the unique critical points of $I_{\lambda}$. Then $I_{\lambda}$ satisfies $(P S)_{c}$, for every $c<I_{\lambda}\left(u_{\lambda}\right)+\frac{2 \pi}{\alpha_{0}}$.

Proof: Let $\left(u_{n}\right) \subset H_{0}^{1}(\Omega)$ be a sequence such that

$$
I_{\lambda}\left(u_{n}\right) \rightarrow c<I_{\lambda}\left(u_{\lambda}\right)+\frac{2 \pi}{\alpha_{0}} \quad \text { and } \quad I_{\lambda}^{\prime}\left(u_{n}\right) \rightarrow 0 .
$$

We observe that $g(x, s) \equiv h(x, s)+\lambda f(x, s)$ satisfies the conditions $\left(h_{2}\right)$ and $(c)_{\alpha_{0}}$. Thus, we may apply Lemma 6.1 from [21] to obtain a subsequence $\left(u_{n_{j}}\right)$ converging weakly in $H_{0}^{1}(\Omega)$ to a solution $u$ of $(3)$ such that

$$
\int_{\Omega} G\left(x, u_{n_{j}}\right) d x \rightarrow \int_{\Omega} G(x, u) d x, \text { as } n \rightarrow \infty,
$$

where $G(x, s)=\int_{0}^{s} g(x, t) d t$. Moreover, we may assume that $u_{n_{j}}(x) \rightarrow u(x)$, for almost every $x \in \Omega$. Then, either $u=0$ or $u=u_{\lambda}$. If $u=0$, using that $I_{\lambda}\left(u_{\lambda}\right)<0$, by (29) and (28) we have

$$
\lim _{n \rightarrow \infty}\left\|u_{n}\right\|^{2}=2\left(c+\int_{\Omega}[H(x, u)+\lambda F(x, u)] d x\right)=2 c<2 I_{\lambda}\left(u_{\lambda}\right)+\frac{4 \pi}{\alpha_{0}}<\frac{4 \pi}{\alpha_{0}} .
$$

Thus, we can choose $q>1$ such that $q \alpha_{0}\left\|u_{n_{j}}\right\|^{2}<4 \pi$, for every $j \geq j_{0}$, for some $j_{0} \in \mathbb{N}$. Consequently, from (23) and Trudinger-Moser inequality, we find

$$
\int_{\Omega}\left|h\left(x, u_{n_{j}}\right)\right|^{q} d x \leq C \int_{\Omega} \exp \left(q \alpha_{0}\left\|u_{n_{j}}\right\|^{2}\left(\frac{u_{n_{j}}}{\left\|u_{n_{j}}\right\|}\right)^{2}\right) d x \leq C .
$$

Since $u_{n_{j}}(x) \rightarrow u(x)$, for almost every $x \in \Omega$, by Egoroff's Theorem, given $\epsilon>0$ there exists $E \subset \Omega$ such that $|E|<\epsilon$ and $u_{n_{j}}(x) \rightarrow u(x)$, uniformly on $x \in \Omega \backslash E$. Observing that

$$
h\left(x, u_{n_{j}}\right) u_{n_{j}}-h(x, u) u=h\left(x, u_{n_{j}}\right)\left(u_{n_{j}}-u\right)+\left(h\left(x, u_{n_{j}}\right)-h(x, u)\right) u,
$$

by Hölder's inequality and (30), we get

$$
\begin{aligned}
& \left|\int_{\Omega}\left(h\left(x, u_{n_{j}}\right) u_{n_{j}}-h(x, u) u\right) d x\right| \leq C\left\|u_{n_{j}}-u\right\|_{L^{q /(q-1)}(\Omega)}+ \\
& \quad+\left|\int_{\Omega \backslash E}\left(h\left(x, u_{n_{j}}\right)-h(x, u)\right) u d x\right|+C \epsilon^{(q-1) / q}
\end{aligned}
$$

As $\epsilon>0$ can be chosen arbitrarily small and $h\left(x, u_{n_{j}}\right) \rightarrow h(x, u)$ uniformly on $\Omega \backslash E$, we have

$$
\int_{\Omega} h\left(x, u_{n_{j}}\right) u_{n_{j}} d x \rightarrow \int_{\Omega} h(x, u) u d x=0
$$

Publicado pelo ICMC-USP Sob a supervisão da $\mathrm{CPq} / \mathrm{ICMC}$ 
Since $f$ has subcritical growth, a similar and more direct argument implies that

$$
\int_{\Omega} f\left(x, u_{n_{j}}\right) u_{n_{j}} d x \rightarrow \int_{\Omega} f(x, u) u d x=0 .
$$

Thus, from (28), we have

$$
\left\|u_{n_{j}}\right\|^{2}=I_{\lambda}^{\prime}\left(u_{n_{j}}\right) u_{n_{j}}+\int_{\Omega} h\left(x, u_{n_{j}}\right) u_{n_{j}} d x+\lambda \int_{\Omega} f\left(x, u_{n_{j}}\right) u_{n_{j}} d x \rightarrow 0 .
$$

Hence $u_{n_{j}} \rightarrow u=0$ strongly in $H_{0}^{1}(\Omega)$.

Now, we suppose that $u=u_{\lambda}$. In this case, by (29),

$$
\left\|u_{n_{j}}\right\|^{2} \rightarrow 2\left(c+\int_{\Omega}\left(H\left(x, u_{\lambda}\right)+\lambda F\left(x, u_{\lambda}\right)\right) d x, \text { as } j \rightarrow \infty .\right.
$$

Taking $v_{j}=\frac{u_{n_{j}}}{\left\|u_{n_{j}}\right\|}$ and defining $d=\int_{\Omega}\left(H\left(x, u_{\lambda}\right)+\lambda F\left(x, u_{\lambda}\right)\right) d x$, we get

$$
v_{j} \rightarrow v=\frac{u_{\lambda}}{(2(c+d))^{1 / 2}}
$$

Consider $\beta>\alpha_{0}$ such that

$$
c<I_{\lambda}\left(u_{\lambda}\right)+\frac{2 \pi}{\beta} .
$$

From $(c)_{\alpha_{0}}$, we find $q>1$ and a constant $C>0$ so that

$$
\max \left\{|f(x, s)|^{q},|h(x, s)|^{q}\right\} \leq C \exp \left(\beta s^{2}\right), \forall x \in \Omega, s \in \mathbb{R} .
$$

Thus, for every $j$,

$$
\max \left\{\int_{\Omega}\left|f\left(x, u_{n_{j}}\right)\right|^{q} d x, \int_{\Omega}\left|h\left(x, u_{n_{j}}\right)\right|^{q} d x\right\} \leq C \int_{\Omega} \exp \left(\beta\left\|u_{n_{j}}\right\|^{2}\left|v_{j}(x)\right|^{2}\right) d x .
$$

On the other hand,

$$
1-\|v\|^{2}<\frac{\frac{4 \pi}{\beta}}{2(c+d)}
$$

Consequently, there exists $p>0$ such that

$$
\frac{\beta}{4 \pi}\left\|u_{n_{j}}\right\|^{2}<p<\frac{1}{1-\|v\|^{2}} .
$$

Hence, by Lemma 3.1 and (31), there exists $M>0$ such that

$$
\max \left\{\int_{\Omega}\left|f\left(x, u_{n_{j}}\right)\right|^{q} d x, \int_{\Omega}\left|h\left(x, u_{n_{j}}\right)\right|^{q} d x\right\} \leq M, \forall j .
$$

Publicado pelo ICMC-USP Sob a supervisão $\mathrm{CPq} / \mathrm{ICMC}$ 
Applying Egoroff's Theorem one more time, the above inequality and the argument employed in the previous case, we obtain

$$
\int_{\Omega}\left[h\left(x, u_{n_{j}}\right) u_{n_{j}}+\lambda f\left(x, u_{n_{j}}\right) u_{n_{j}}\right] d x \rightarrow \int_{\Omega}\left[h\left(x, u_{\lambda}\right) u_{\lambda}+\lambda f\left(x, u_{\lambda}\right) u_{\lambda}\right] d x,
$$

as $j \rightarrow \infty$. Therefore, by (29) and using that $u_{\lambda}$ is a critical point of $I_{\lambda}$, we get

$$
\left\|u_{n_{j}}\right\|^{2} \rightarrow \int_{\Omega}\left[h\left(x, u_{\lambda}\right) u_{\lambda}+\lambda f\left(x, u_{\lambda}\right) u_{\lambda}\right] d x=\left\|u_{\lambda}\right\|^{2} .
$$

The lemma is proved.

In order to find the appropriate level to the use of Mountain Pass Theorem we follow the method used in $[1,2,14,21]$. Before to state our estimate, we define the Moser functions (see [18]). Let $x_{0} \in \Omega$ and $R>0$ such that $B_{R}\left(x_{0}\right) \subset \Omega$. The Moser functions are defined for $0<r<R$ by

$$
M_{r}(x)=\frac{1}{\sqrt{2 \pi}}\left\{\begin{array}{cl}
\left(\log \frac{R}{r_{R}}\right)^{\frac{1}{2}} & \text { if } 0 \leq\left|x-x_{0}\right| \leq r \\
\frac{\log \frac{x-x_{0} \mid}{\left.\mid x-\frac{R}{r}\right)^{\frac{1}{2}}}}{(\log } r \leq\left|x-x_{0}\right| \leq R \\
0 & \text { if }\left|x-x_{0}\right| \geq R
\end{array}\right.
$$

Then, $M_{r} \in H_{0}^{1}(\Omega),\left\|M_{r}\right\|=1$ and $\operatorname{supp}\left(M_{r}\right) \subset B_{R}\left(x_{0}\right)$.

Let $r>0$ be given by Lemma 2.2 for $\gamma=3$. Since $u_{\lambda}>0$ and $u_{\lambda} \in C^{1}(\bar{\Omega})$, we obtain $x_{0} \in \Omega$ and $R, r_{0}>0$ such that $B_{R}\left(x_{0}\right) \subset \Omega$ and

$$
0<r_{0} \leq u_{\lambda}(x) \leq r, \forall x \in B_{R}\left(x_{0}\right)
$$

We Consider the Moser sequence $M_{n}=M_{\frac{R}{n}}$, for every $n \in \mathbb{N}$.

Taking $\lambda^{*}>0$ and $u_{\lambda}$, for $0<\lambda<\lambda^{*}$, given in the proof of Proposition 2.1, we have

Proposition 3.2. Suppose $h \in C(\bar{\Omega} \times \mathbb{R}, \mathbb{R})$ satisfies $\left(h_{1}\right)-\left(h_{4}\right)$ and $(c)_{\alpha_{0}}$, with $\alpha_{0}>0$, and $f \in C(\bar{\Omega} \times \mathbb{R}, \mathbb{R})$ satisfies $\left(f_{3}\right)-\left(f_{4}\right)$ and $(c)_{\alpha_{0}}$, with $\alpha_{0}=0$. Then, for every $\lambda \in\left(0, \lambda^{*}\right)$, there exists $n \in \mathbb{N}$ satisfying

$$
\max \left\{I_{\lambda}\left(u_{\lambda}+t M_{n}\right) ; t \geq 0\right\}<I_{\lambda}\left(u_{\lambda}\right)+\frac{2 \pi}{\alpha_{0}} .
$$

The proof of Proposition 3.2 will be carried out through the verification of several steps. First, we suppose by contradiction that, for every $n \in \mathbb{N}$, we have

$$
\max \left\{I_{\lambda}\left(u_{\lambda}+t M_{n}\right) ; t \geq 0\right\} \geq I_{\lambda}\left(u_{\lambda}\right)+\frac{2 \pi}{\alpha_{0}} .
$$

Publicado pelo ICMC-USP Sob a supervisão da $\mathrm{CPq} / \mathrm{ICMC}$ 
From $(h)_{\alpha_{0}}$ and $\left(h_{2}\right)$,

$$
\lim _{t \rightarrow \infty} I_{\lambda}\left(u_{\lambda}+t M_{n}\right)=-\infty
$$

Thus, there exists $t_{n}>0$ such that

$$
I_{\lambda}\left(u_{\lambda}+t_{n} M_{n}\right)=\max \left\{I_{\lambda}\left(u_{\lambda}+t M_{n}\right) ; t \geq 0\right\} .
$$

Lemma 3.3. There are $n_{0} \in \mathbb{N}$ and a positive constant $C=C\left(\lambda, n_{0}\right)$ such that

$$
t_{n}^{2} \geq \frac{4 \pi}{\alpha_{0}}-\frac{C t_{n}^{3}}{(\log n)^{3 / 2}} \forall n \geq n_{0} .
$$

Proof: From (33) and (35),

$$
I_{\lambda}\left(u_{\lambda}\right)+\frac{2 \pi}{\alpha_{0}} \leq I_{\lambda}\left(u_{\lambda}+t_{n} M_{n}\right) .
$$

Using that $u_{\lambda}$ is a solution of (3), we get

$$
\begin{aligned}
\frac{2 \pi}{\alpha_{0}} & \leq I_{\lambda}\left(u_{\lambda}+t_{n} M_{n}\right)-I_{\lambda}\left(u_{\lambda}\right) \\
& =\frac{t_{n}^{2}}{2}-\int_{\Omega}\left(H\left(x, u_{\lambda}+t_{n} M_{n}\right)-H\left(x, u_{\lambda}\right)-h\left(u_{\lambda}\right) t_{n} M_{n}\right) d x \\
& -\lambda \int_{\Omega}\left(F\left(x, u_{\lambda}+t_{n} M_{n}\right)-F\left(x, u_{\lambda}\right)-f\left(x, u_{\lambda}\right) t_{n} M_{n}\right) d x
\end{aligned}
$$

From $\left(f_{4}\right),\left(h_{4}\right)$, and $(40)$, using Lemma 2.2 , we have

$$
\frac{2 \pi}{\alpha_{0}} \leq \frac{t_{n}^{2}}{2}+C \int_{\Omega} t_{n}^{3} M_{n}^{3} d x
$$

On the other hand, from definition of $M_{n}$, we find

$$
\int_{\Omega} M_{n}^{3}(x) d x \leq \frac{C}{(\log n)^{3 / 2}}
$$

for some positive constant $C$. Using (36) and (37), we conclude the proof of the lemma.

As consequence of Lemma 3.3 we conclude that the sequence $\left(t_{n}\right)$ does not have any convergent subsequence to zero.

Lemma 3.4. The sequence $\left(t_{n}^{2}\right)$ converges to $4 \pi / \alpha_{0}$.

Publicado pelo ICMC-USP Sob a supervisão $\mathrm{CP} q / \mathrm{ICMC}$ 
Proof: Since $\frac{d}{d t} I_{\lambda}\left(u_{\lambda}+t M_{n}\right)=0$ for $t=t_{n}$, we have

$$
\int_{\Omega} \nabla\left(u_{\lambda}+t_{n} M_{n}\right) \nabla\left(t_{n} M_{n}\right) d x=\int_{\Omega}\left(h\left(x, u_{\lambda}+t_{n} M_{n}\right)+\lambda f\left(x, u_{\lambda}+t_{n} M_{n}\right) t_{n} M_{n} d x .\right.
$$

Since $h$ and $f$ are continuous and $h$ satisfies $\left(h_{3}\right)$ and $\left(c_{\alpha_{0}}\right)$, with $\alpha_{0}>0$, and $f$ satisfies $\left(c_{\alpha_{0}}\right)$, with $\alpha_{0}=0$, given $K>0$ there exists a constant $C>0$ such that

$$
s[h(x, s)+\lambda f(x, s)] \geq K \exp \left(\alpha_{0} s^{2}\right)-C, \forall s \geq 0, x \in \Omega .
$$

Hence, from Hölder's inequality, we have

$$
t_{n}\left\|u_{\lambda}+t_{n} M_{n}\right\| \geq K \int_{B_{R}\left(x_{0}\right)}\left[\exp \left(\alpha_{0}\left|u_{\lambda}+t_{n} M_{n}\right|^{2}\right)-C\right] \frac{t_{n} M_{n}}{u_{\lambda}+t_{n} M_{n}} d x .
$$

Using that $0<r_{0} \leq u_{\lambda}(x) \leq r$, for every $x \in B_{R}\left(x_{0}\right)$, and the definition of $M_{n}$, we find

$$
\begin{aligned}
t_{n}\left\|u_{\lambda}+t_{n} M_{n}\right\| & \geq K \int_{B_{\frac{R}{n}\left(x_{0}\right)}} \exp \left(\alpha_{0} t_{n}^{2} \frac{\log n}{2 \pi}\right) \frac{t_{n}\left(\frac{\log n}{2 \pi}\right)^{1 / 2}}{r+t_{n}\left(\frac{\log n}{2 \pi}\right)^{1 / 2}} d x \\
& -C \int_{B_{R}\left(x_{0}\right)} \frac{t_{n} M_{n}}{u_{\lambda}+t_{n} M_{n}} d x .
\end{aligned}
$$

Since $\left(t_{n}\right)$ does not converge to zero and $\log n \rightarrow \infty$, as $n \rightarrow \infty$, there is $n_{0} \in \mathbb{N}$ such that

$$
\begin{aligned}
t_{n}\left\|u_{\lambda}+t_{n} M_{n}\right\| & \geq \frac{K \pi R^{2}}{2 n^{2}} \exp \left(\alpha_{0} t_{n}^{2} \frac{\log n}{2 \pi}\right)-C \int_{B_{R}\left(x_{0}\right)} \frac{t_{n} M_{n}}{u_{\lambda}+t_{n} M_{n}} d x \\
& \geq \frac{K \pi R^{2}}{2} \exp \left[\left(\frac{\alpha_{0}}{4 \pi} t_{n}^{2}-1\right) 2 \log n\right]-C \int_{B_{R}\left(x_{0}\right)} \frac{t_{n} M_{n}}{u_{\lambda}+t_{n} M_{n}} d x,
\end{aligned}
$$

for every $n \geq n_{0}$. Moreover, as $0<r_{0} \leq u_{\lambda}(x)$, for every $x \in B_{R}\left(x_{0}\right)$, we can write

$$
t_{n}\left\|u_{\lambda}+t_{n} M_{n}\right\| \geq K \pi \frac{R^{2}}{2} \exp \left[\left(\frac{\alpha_{0}}{4 \pi} t_{n}^{2}-1\right) 2 \log n\right]-C \pi R^{2}, \forall n \geq n_{0} .
$$

This implies that $\left(t_{n}\right)$ is a bounded sequence. Now, we observe

$$
\begin{aligned}
\int_{B_{R}\left(x_{0}\right)} \frac{t_{n} M_{n}}{u_{\lambda}+t_{n} M_{n}} d x= & \int_{B_{\frac{R}{n}}\left(x_{0}\right)} \frac{t_{n} M_{n}}{u_{\lambda}+t_{n} M_{n}} d x+\int_{B_{R}\left(x_{0}\right) \backslash B_{\frac{R}{n}}\left(x_{0}\right)} \frac{t_{n} M_{n}}{u_{\lambda}+t_{n} M_{n}} d x \\
& \leq \pi \frac{R^{2}}{n^{2}} \frac{t_{n}\left(\frac{\log n}{2 \pi}\right)^{1 / 2}}{r_{0}+t_{n}\left(\frac{\log n}{2 \pi}\right)^{1 / 2}}+\int_{B_{R}\left(x_{0}\right) \backslash B_{\frac{R}{n}}(0)} \frac{t_{n} \log \left(\frac{R}{|x|}\right)}{(\log n)^{1 / 2}} d x
\end{aligned}
$$

Thus, from Lebesgue's dominated convergence theorem and using that $\left(t_{n}\right)$ is bounded, we have

$$
\int_{B_{R}\left(x_{0}\right)} \frac{t_{n} M_{n}}{u_{\lambda}+t_{n} M_{n}} d x \rightarrow 0, \text { as } n \rightarrow \infty .
$$


Combining (38) with (40), we obtain $t_{n}^{2} \rightarrow \frac{4 \pi}{\alpha_{0}}$. This concludes the proof of the lemma.

Now, we are ready to conclude the proof of Proposition 3.2. By Lemma 3.3 and Lemma 3.4 , there is a constant $C>0$ such that

$$
t_{n}^{2} \geq \frac{4 \pi}{\alpha_{0}}-\frac{C}{(\log n)^{3 / 2}}, \forall n \geq n_{0} .
$$

From (38),

$$
t_{n}\left\|u_{\lambda}+t_{n} M_{n}\right\| \geq \frac{K R^{2} \pi}{2} \exp \left(-\frac{C \alpha_{0}}{2 \pi \sqrt{\log n}}\right)-C \int_{B_{R}\left(x_{0}\right)} \frac{t_{n} M_{n}}{u_{\lambda}+t_{n} M_{n}} d x
$$

for every $n \geq n_{0}$. Then, taking $n \rightarrow \infty$ and using (40) we obtain

$$
2\left\|u_{\lambda}\right\| \sqrt{\frac{\pi}{\alpha_{0}}}+\frac{4 \pi}{\alpha_{0}} \geq \frac{K R^{2} \pi}{2} .
$$

However, this gives us a contradiction, because $K$ can be chosen arbitrarily large. The proof of Proposition 3.2 is complete.

Now, we may conclude the proof of Theorem 1.2. Arguing by contradiction, we suppose that zero and $u_{\lambda}$ are the only possible solutions of (3). By Lemma 3.2, $I_{\lambda}$ satisfies $(P S)_{c}$ for every $c<I_{\lambda}\left(u_{\lambda}\right)+2 \pi / \alpha_{0}$. Furthermore, by (25) and (34), $I_{\lambda}$ satisfies the geometric hypotheses of the Mountain Pass Theorem [4]. Invoking Proposition 3.2 and using that $I_{\lambda}\left(u_{\lambda}\right)<0$, we conclude that $I_{\lambda}$ possesses at least three critical points. However, this contradicts the fact that zero and $u_{\lambda}$ are the only possible critical points of $I_{\lambda}$. Theorem 1.2 is proved.

\section{REFERENCES}

1. Adimurthi, "Existence of positive solutions of the semilinear Dirichlet problems with critical growth for the N-Laplacian", Ann. Sc. Norm. Sup. Pisa Cl. Sci. 17, 393-413, 1990.

2. Adimurthi, Yadava, S. L., "Multiplicity results for semilinear elliptic equations in a bounded domain of $\mathbb{R}^{2}$ involving critical exponent", Ann. Sc. Norm. Sup. Pisa Cl. Sci. 17, 481-504, 1990.

3. Ambrosetti, A., Brezis, H., Cerami, G., "Combined effects of concave and convex nonlinearities in some elliptic problems", J. Func. Anal. 122, 519-543, 1994.

4. Ambrosetti, A., Rabinowitz, P. H., "Dual variational methods in critical point theory and applications", J. Func. Anal. 14 349-381, 1973.

5. Arcoya, D., Carmona, J., Pellacci, B., "Bifurcation for some quasilinear operators", Proc. Roy. Soc. Edinb. 131A, 733-765, 2001.

6. Bartsch, T., Willem, M., "On an elliptic equation with concave and convex nonlinearities", Proc. Amer. Math. Soc. 123, 3555-3561, 1995.

7. Bezerra do Ó, J. M., "Quasilinear elliptic equations with exponential nonlinearities", Comm. Appl. Nonlinear Anal. 3, 63-72, 1995.

8. Boccardo, L., Escobedo, M., Peral Alonso, I., "A Dirichlet problem involving critical exponent", Nonlinear Anal. 24, 1639-1648, 1995. 
9. Brezis, H., Lieb, E., "A relation between pointwise convergence of functions and convergences of functionals", Proc. Amer. Math. Soc. 88, 486-490, 1983.

10. Brezis, H., Nirenberg, L., "Positive solutions of nonlinear elliptic equations involving critical exponents", Comm. Pure Appl. Math. 36, 437-477, 1983.

11. DiBenedetto, E., " $C^{1, \alpha}$ local regularity of weak solutions of degenerate elliptic equations", Nonlinear Analysis-TMA 8, 827-850, 1983.

12. Drábek, P., Huang, Y. X., "Multiplicity of positive solutions for some quasilinear elliptic equation in $\mathbb{R}^{N}$ with critical Sobolev exponent", J. Diff. Eq. 140, 106-132, 1997.

13. Ekeland, I., "On the variational principle", J. Math. Anal. Applic. 47, 324-353, 1974.

14. Figueiredo, D. G. de, Miyagaki, O. H., Ruf, B., "Elliptic equations in $\mathbb{R}^{2}$ with nonlinearities in the critical growth range", Calc. Var. 3, 139-153, 1995.

15. Garcia Azorero, J. P., Peral Alonso, I., Manfredi, J. J., "Sobolev versus Hölder local minimizers and global multiplicity for some quasilinear elliptic equations", Commun. Contemp. Math. 2, 385-404, 2000.

16. Garcia Azorero, J., Peral Alonso, I., "Some results about the existence of a second positive solution in a quasilinear critical problem", Ind. Univ. Math. J. 43, 941-957, 1994.

17. Gilbarg, D., Trudinger, S., "Elliptic Partial Differential Equations of Second Order", Springer-Verlag, Berlin Heidelberg, 1983.

18. Moser, J., "A sharp form of an inequality by N. Trudinger", Ind. Univ. Math. J. 20, 185-201, 1985.

19. Lions, P. L., "Concentration-compactness principle in the calculus of variations. The limit case, part 1", Rev. Mat. Iberoamericana 1, 145-283, 1985.

20. Rabinowitz, P. H., "Minimax methods in critical point theory with applications to differential equations", CBMS Regional Conf. Ser. in Math. 65, AMS, Providence, RI, 1986.

21. Silva, E. A. B., Soares, S. H. M., "Liouville-Gelfand type problem for the N-laplacian on bounded

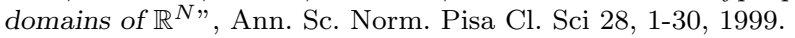

22. Trudinger, N., "On embedding into Orlicz spaces and some applications", J. Math. Mech. 17, 473-484, 1967.

23. Wang, Z. Q., "Nonlinear boundary value problems with concave nonlinearities near the origin", NoDEA 8, 15-33, 2001. 